\title{
Renale Denervation bei nicht-kontrollierter arterieller Hypertonie
}

\author{
Gemeinsame Stellungnahme der Deutschen Hochdruckliga, Deutschen Gesellschaft für \\ Kardiologie / Herz- und Kreislaufforschung, Deutschen Gesellschaft für Angiologie / Gefäß- \\ medizin, Deutschen Gesellschaft für Nephrologie und Deutschen Röntgen Gesellschaft
}

\begin{abstract}
Die Hypertonie ist einer der Hauptrisikofaktoren für kardiovaskuläre Morbidität und Sterblichkeit - das ist bekannt und hinlänglich gesichert [1]. Dennoch erreichen in Deutschland zu wenig Patienten mit Bluthochdruck die empfohlenen Zielwerte. Interventionelle Therapien sollten in diesen Fällen nur nach sehr engen Regeln eingesetzt werden - so dieses Positionspapier.
\end{abstract}

Die renale Denervation ist ein interventionelles Verfahren zur Verödung efferenter sympathischer und afferenter sensorischer Nervenfasern in der Adventitia der Nierenarterien. Die ersten Studienergebnisse zur Blutdrucksenkung mit diesem Verfahren wurden 2009 veröffentlicht [2]. Es folgten Stellungnahmen nationaler und internationaler Fachgesellschaften [3-5]. Die bisher vorliegenden Studienergebnisse, die eine signifikante Blutdrucksenkung belegen, stammen aus ausgewählten europäischen Zentren; alle Patienten wurden wissenschaftlich nachverfolgt.

Die im März 2014 publizierte Zulassungsstudie Symplicity HTN-3 - gefördert von der US-amerikanischen Federal Drug Administration (FDA) - erreichte den primären Sicherheitsendpunkt von periprozeduralen und vaskulären Komplikationen nach 6 Monaten. Jedoch wurde der primäre Effektivitätsendpunkt - Senkung des Gelegenheitsblutdruckes - verfehlt [6]. Die Autoren der Studie haben mittlerweile methodische Schwächen eingeräumt [7]; diese wurden an anderer Stelle bereits ausführlich diskutiert [8-10] und auch die European Society of Hypertension [11] sowie die Deutsche Gesellschaft für Kardiologie haben in ihren Stellungnahmen darauf hingewiesen [8].

Der aktuelle deutsche Kommentar [12] zur ESH/ ESC-Leitlinie 2013 zum Management der arteriellen Hypertonie, der nach Veröffentlichung der HTN3-Studie erschienen ist, besagt: Ein invasives Verfahren wie die renale Denervation kann erst in Betracht gezogen werden, wenn unter einer maximalen medikamentösen Behandlung die Zielwerte nicht erreicht werden [12].

Es wird empfohlen, die invasiven Therapien nur bei wirklich therapieresistentem Bluthochdruck anzuwenden:

- systolisch $\geq 160 \mathrm{mmHg}$ oder

- diastolisch $\geq 110 \mathrm{mmHg}$ und
- nach Ausschluss einer Pseudoresistenz durch Langzeitblutdruckmessung.

Bis Beweise für eine langfristige Wirksamkeit und Sicherheit der renalen Denervation vorliegen, wird empfohlen, dass

- Diagnostik und Nachverfolgung auf spezialisierte Zentren beschränkt bleiben und

- invasive Verfahren von erfahrenen Interventionalisten durchgeführt werden (Definition spezialisiertes Zentrum: [3]).

Hieran haben nach unserer Ansicht die Ergebnisse von Symplicity HTN-3 nichts geändert (s.o.).

Die behandelten Patienten müssen vollständig in einem - möglichst Industrie-unabhängigen - Register gemeldet werden. Die renale Denervation muss in randomisierten, kontrollierten Studien in Deutschland weiter kritisch evaluiert werden, um den Stellenwert des Verfahrens abschließend beurteilen zu können. Renale Denervation ist keine Alternative zu einer etablierten medikamentösen und nicht medikamentösen Therapie. Vielmehr ist sie bei konservativ therapierefraktären Patienten im Einzelfall als zusätzliches Verfahren zu sehen. Die Entscheidung über die Indikationsstellung auf Basis klarer Kriterien muss ärztliche Aufgabe sein und bleiben.

\section{Interessenkonflikt}

O. V.: Vortragshonorare und/ oder Beratertätigkeit für Medtronic, St. Jude und Boston Scientific; M. B. und F. M.: Studienunterstützung, Vortragshonorare und/ oder Beratertätigkeit für Medtronic, St. Jude und Cordis; S. E.: Vortragshonorare von St. Jude Medical; H. R.: keine; L-C.R.: Vortragshonorare und Beratertätigkeit für Medtronic; R. S.: Vortragshonorare und Beratertätigkeit für Boston Scientific, Kona Medical, Medtronic, Recor, Terumo und Medtronic; M.U.: Vortragshonorare von Medtronic J.W.: Vortragshonorare von Medtronic und ReCor

Vollständiges Literaturverzeichnis unter http://dx.doi.org/10.1055/s-0041-100835

\section{Autoren}

Oliver Vonend,

Michael Böhm,

Siegfried Eckert,

Martin Hausberg,

Harald Rittger,

Lars-Christian Rump,

Roland Schmieder,

Karl-Ludwig Schulte,

Heribert Schunkert,

Michael Uder,

Roland Veelken,

Dierk Vorwerk,

Joachim Weil,

Ulrich Wenzel,

Felix Mahfoud

\section{Korrespondenz}

Prof. Dr. med. Oliver Vonend PD Dr. med. Felix Mahfoud Sprecher der Kommission Interventionelle Hochdrucktherapie

Deutsche Hochdruckliga e. V. $\mathrm{DHL}^{\circledR}$; Deutsche Gesellschaft für Hypertonie und Prävention Berliner Straße 46

69120 Heidelberg Vonend@nephrologiewiesbaden.de

Felix.Mahfoud@uniklinikumsaarland.de

DOI 10.1055/s-0041-100835 Dtsch Med Wochenschr 2015; 140: 363

(c) Georg Thieme Verlag KG . Stuttgart - New York . ISSN 0012-0472 


\section{Literatur}

1 Mancia G, Fagard R, Narkiewicz K et al. 2013 ESH/ESC Practice Guidelines for the Management of Arterial Hypertension: The Task Force for the Management of Arterial Hypertension of the European Society of Hypertension (ESH) and of the European Society of Cardiology (ESC). Eur Heart J 2013; 34: 2159-2219

2 Krum H, Schlaich M, Whitbourn R et al. Catheter-based renal sympathetic denervation for resistant hypertension: a multicentre safety and proof-of-principle cohort study. Lancet 2009; 373: 1275-1281

3 Mahfoud F, Vonend O, Bruck $\mathrm{H}$ et al. [Expert consensus statement on interventional renal sympathetic denervation for hypertension treatment]. Dtsch Med Wochenschr 2011; 136: 2418

4 Schmieder RE, Redon J, Grassi G et al. ESH position paper: renal denervation - an interventional therapy of resistant hypertension. J Hypertens 2012; 30: 837-841

5 Mahfoud F, Lüscher TF, Andersson B et al. Expert consensus document from the European Society of Cardiology on catheter-based renal denervation. Eur Heart J 2013; 34: 2149-2157

6 Bhatt DL, Kandzari DE, O‘Neill WW et al. A controlled trial of renal denervation for resistant hypertension. $\mathrm{N}$ Engl J Med 2014; 370: 1393-1401
7 Kandzari DE, Bhatt DL, Brar S et al. Predictors of blood pressure response in the Symplicity HTN-3 trial. Eur Heart J 2015; 36: 219-227

8 Böhm M, Hamm CW, - Kuck KH et al. Stellungnahme der Deutschen Gesellschaft für Kardiologie zu der Symplicity HTN-3-Studie bei Patienten mit resistenter arterieller Hypertonie nach renaler Denervation. Kardiologe, 2014; 8: 244-245

9 Lüscher TF, Mahfoud F. Renal nerve ablation afte SYMPLICITY HTN-3: confused at the higher level? Eur Heart ] 2014; 35: 1706-1711

10 Schmieder RE Hypertension: How should data from SYMPLICITY HTN-3 be interpreted? Nat Rev Cardiol 2014; 11: 375-376

11 European Society of Hypertension. ESH Statement on SYMPLICITY HTN-3 Results. www.eshonline.org/asset. axd?id=45225f42-8911-4b76-8c84-99c993be3813 $\& \mathrm{t}=635415226410030000$, Stand 10.2.2015

12 Kintscher U, Böhm M, Goss F. et al. Kommentar zur 2013-ESH/ESC-Leitlinie zum Management der arteriellen Hypertonie. Kardiologe 2014; 8: 223-230 